\title{
LA GESTIÓN DEL CONOCIMIENTO, FACTOR ESTRATÉGICO PARA EL DESARROLLO
}

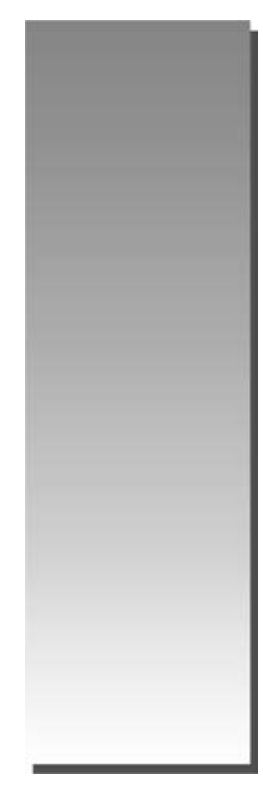

Milagro Rodríguez Andino (*)

E-mail:milagro.rodriguez@reduc.edu.cu

Fernando García Colina (**)

E-mail: fernando.garcia@reduc.edu.cu

Miguel Ángel Pérez Hernández ( ${ }^{* * * * *}$

E-mail: miguel.perez@reduc.edu.cu

Juan Victoriano Castillo Maza ( ${ }^{* * * * * *)}$

E-mail: jcastillom@unmsm.edu.pe

\section{RESUMEN}

La gestión del conocimiento ha adquirido una importancia significativa como factor de cambio y desarrollo en todo el quehacer de la sociedad. Su principal misión es crear un ambiente en el que el conocimiento y la información disponibles en una organización sean accesibles y puedan ser usados para estimular la innovación, provocar mejoras en la toma de decisiones y producir nuevos conocimientos. La clave está en crear una cultura donde la información y el conocimiento se valoren, se compartan, se gestionen y se usen eficaz y eficientemente.

Las Tecnologías de la Información y las Comunicaciones (TIC) han proporcionado herramientas de indudable valor para la generación de bases de conocimiento, y constituyen un nuevo ámbito de investigación y desarrollo en este sentido.

(*) Doctora en Ciencias de la Educación por la Universidad de La Habana. Profesora Titular de la Facultad de Ciencias Económicas y Empresariales (Universidad de Camagüiey).

${ }^{(* * *)}$ Doctor en Ciencias Pedagógicas. Profesor Titular de Técnicas de Dirección en la Facultad de Ciencias Económicas y Empresariales (Universidad de Camaguiey).

(***) Doctor en Ciencias Pedagógicas. Profesor Titular y Director del Centro de Post Grado Internacional (Universidad de Camagiiey).

(*****) Doctor en Ciencias Contables y Empresariales, Magíster en Economía con mención en Métodos Cuantitativos y Licenciado en Administración. Profesor Principal y Director de la Unidad de Post Grado de la Facultad de Ciencias Administrativas de la UNMSM. Profesor de Post Grado en UNFV, UNASAM, UNSCH, UNEGV, UNJBG, UNSAAC, UNP, UNCP, UNA, UCV, UANCV. 
El trabajo que presentamos pretende valorar importantes aspectos a atender; entre éstos tenemos la política y los elementos principales de la estrategia para gestionar el conocimiento con el empleo de las TIC, que permita impulsar el desarrollo de una organización.

Palabras Clave: Gestión del Conocimiento y Desarrollo, TIC y Gestión del Conocimiento, TIC e Inteligencia Empresarial.

\begin{abstract}
Management of the knowledge has acquired a meaningful importance as change and development factor in all the occupation of the society. The principal mission of this is to create an environment in the one which the knowledge and the available information in an organization will be accessible and they could be used to stimulate the innovation, to provoke improvements in the decision making and to produce new knowledge, the key is in creating a culture in the one which the information and the knowledge are valued, are shared, are transacted and are used effective and efficiently.

The Information Technology and Communications (TIC) have come to provide tools of certain value for the generalization of knowledge bases and constitute a new research and development area in this sense.

The work that we present intends to value important aspects to attend between these the policy and the principal elements of the strategy to reach management of the knowledge with the employment of the TIC that permit to impel the development of an organization.
\end{abstract}

Key Words: Knowledge and Development Management, TIC and Administration of Knowledge, TIC and Managerial Intelligence.

\section{INTRODUCCIÓN}

El siglo XXI se caracteriza por ser la era del conocimiento. Los activos intangibles (recursos humanos, propiedad intelectual, etc.) han pasado a desempeñar un papel importante en la economía de las empresas, convirtiéndose el conocimiento en un elemento fundamental para la competitividad y el desarrollo económico.

La gestión del conocimiento (GC) ha adquirido, por tanto, una importancia significativa como factor de cambio y desarrollo en todo el quehacer de la sociedad. Su principal misión es crear un ambiente en el que el conocimiento y la información disponibles en una organización sean accesibles y puedan ser usados para estimular la innovación, provocar mejoras en la toma de decisiones y producir nuevos conocimientos; la clave está en crear una cultura en la que la información y el conocimiento se valoren, se compartan, se gestionen y se usen eficaz y eficientemente.

Las últimas investigaciones, estudios y artículos acerca de la GC mencionan la importancia de herramientas que faciliten la captura, conservación, organización y procesamiento y, sobre todo, la difusión del conocimiento de tal forma que se transforme dicho recurso, hoy disperso, en capital intelectual útil a las organizaciones para alcanzar un desarrollo sostenible. Las Tecnologías de la In- formación y las Comunicaciones (TIC) han venido a proporcionar esas herramientas de indudable valor para la generalización de bases de conocimiento y, en este sentido, constituyen un nuevo ámbito de investigación y desarrollo.

En cada uno de los procesos que conforman la actividad de una entidad o empresa es necesario incorporar la dimensión de la gestión de conocimiento, donde se tengan en cuenta acciones y métodos para la recopilación, almacenamiento, generación y distribución del conocimiento; de esta forma se logra que la GC forme parte de toda la entidad como componente fundamental para la consecución de los objetivos que se definan, y para incrementar el capital intelectual de la misma.

El trabajo que presentamos pretende valorar importantes aspectos a atender, entre éstos se encuentran la política y los elementos principales de la estrategia para gestionar el conocimiento con el empleo de las TIC, que permita impulsar el desarrollo de una organización.

\section{DESARROLLO}

Los progresivos cambios generados por la llamada revolución tecnológica, cuyos ejes fundamentales fueron la innovación en todos los campos de la ciencia y en particular en las TIC, no solo han determinado cambios multidimen- 
sionales sino que han dado origen a un nuevo paradigma social y a una nueva cultura.

Surgen así los términos: "Sociedad de la Información" y "Sociedad del Conocimiento". La primera es entendida como el conjunto de relaciones sociales de cualquier tipo que se establecen usando como medio y soporte las TIC, especialmente las redes telemáticas; en este caso, la información es el factor clave que se transforma en un bien de consumo, en un elemento accesible que da poder, indispensable para ser competitivo en un mundo globalizado.

La Sociedad del Conocimiento es un concepto más amplio que hace referencia a la importancia creciente que la ciencia y la tecnología tienen para la creación de riqueza y desarrollo económico. Es en este ámbito que el conocimiento ha adquirido particular relevancia, ya que se convierte en un factor estratégico que permite dar respuesta a estímulos como resultado de muchas razones en un escenario dinámico y en permanente cambio.

Esta etapa marca el inicio de una nueva cultura, con nuevos valores, nuevos requerimientos de capacidades y destrezas, nuevas instituciones y el surgimiento de nuevas carreras y profesionales. En este contexto de profundos cambios impulsados por las TIC es importante partir de la conceptualización y diferenciación de los términos "dato", "información" y "conocimiento". No se trata de sinónimos sino de conceptos que aunque interconectados e interdependientes son diferentes ${ }^{1}$ :

- Dato: Es una representación formalizada de entidades o hechos de carácter simbólico, consecuentemente adecuada para su comunicación, interpretación y procesamiento mediante medios humanos y automáticos. Representan observaciones o hechos fuera de contexto y, por lo tanto, sin significado inmediato. Es la materia prima de la información y no conlleva un significado inherente, sólo indica que ha pasado sin aportar elementos para formarse un juicio ni para elaborar una interpretación o una base de acción sustentable.

- Información: Es el significado que una persona asigna a un dato, lo que implica que el dato se transforma en información cuando es evaluado para alcanzar un objetivo específico. Un dato se transforma en información cuando se le agrega valor, es decir, cuando se contextualiza, se relaciona a un propósito, se categoriza, se procesa, se corrige o se comprime.

- Conocimiento: Es lo que llegamos a crear y a valorar a partir de la información significativa, mediante el agregado de experiencia, comunicación e inferencia. El conocimiento ocurre dentro de y entre individuos. Por estar tan ligado a los seres humanos resulta complejo administrarlo, transferirlo o compartirlo.

"Para las organizaciones el conocimiento representa un activo intangible y estratégico que genera ventajas competitivas. Las organizaciones orientadas hacia los servicios como es el caso de las universidades, se basan en el conocimiento.

Su competitividad está determinada por la inteligencia colectiva, su capital intelectual se constituye en la fuente clave para su diferenciación"'.

Según el modelo de Nonaka y Takeuchi (1995) existen cuatro formas de conversión de conocimiento que surgen cuando el conocimiento tácito y el explícito interactúan, proceso dinámico que hace que la creación del conocimiento se desarrolle a través de un ciclo continuo y acumulativo de generación, codificación y transferencia del conocimiento, la llamada "espiral de creación del conocimiento". En el Gráfico № 1 se observa su funcionamiento.

\section{Gráfico No 1 \\ ESPIRAL DE CREACIÓN DEL CONOCIMIENTO}

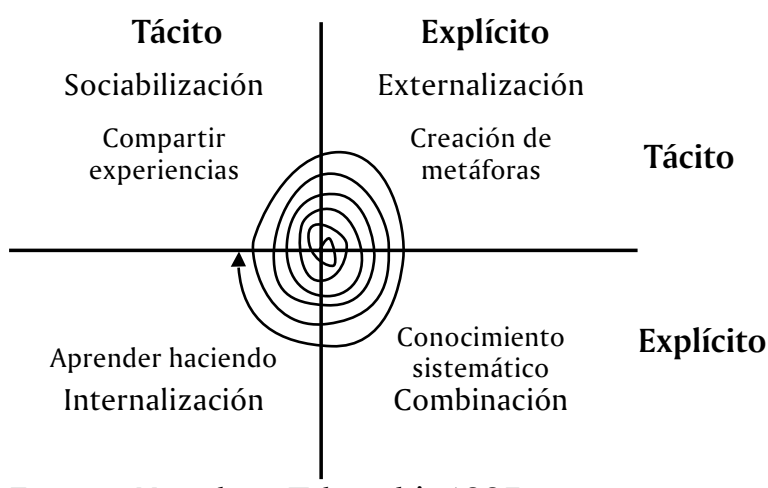

Fuente: Nonaka y Takeuchi, 1995.

1 Saroka, R. (2001). Págs. 4-5.

2 Darín, S. (2006). 
En la actualidad existe consenso en cuanto a que el conocimiento es el recurso más importante de una organización; esto ha derivado en el surgimiento de la gestión del conocimiento como concepto o disciplina científica. Tres razones amparan este fenómeno:

- Necesidad: Las organizaciones de hoy tienen que procesar mayor cantidad de información para tomar decisiones y dar respuestas en tiempos cada vez menores.

- Reconocimiento de la necesidad: Las organizaciones reconocen cada vez más la necesidad e importancia de gestionar el conocimiento.

- Disponibilidad de herramientas para la GC: Cada día aparecen más y más herramientas informáticas para la GC en las organizaciones.

Partiendo de estos tres elementos podemos entonces analizar algunas definiciones de gestión del conocimiento.

"La Gestión del Conocimiento es todo el conjunto de actividades realizadas con el fin de utilizar, compartir y desarrollar los conocimientos de una organización y de los individuos que en ella trabajan, encaminándolos a la mejor consecución de sus objetivos"3.

"La Gestión del Conocimiento es el proceso sistémico y organizacional específico para la adquisición, organización y comunicación del conocimiento de los empleados, de tal forma que los mismos puedan hacer uso de este para ser más efectivos y productivos en su trabajo"4.

La gestión del conocimiento da respuesta al problema de cómo administrar los intangibles de la organización en función de incrementar el valor agregado y la competitividad, mediante la innovación y perfeccionamiento continuo. Se consideran intangibles:

- Capital Humano: Capacidades, limitaciones y actitudes del personal.

- Capital Organizativo: Tecnología, estrategia, cultura, estructuras, procedimientos, etc.

- Capital Relacional: Integrado por el conjunto de relaciones de la organización con clientes, proveedores, distribuidores, socios, etc.
La gestión del conocimiento pretende optimizar la creación y el uso del conocimiento para obtener valor. Dentro del proceso de gestión se desarrollan en forma de ciclo un conjunto de componentes que se conocen como funciones de gestión, y ellas están asociadas a la planificación, la organización, la dirección y el control.

Las entidades que se dedican a la gestión del conocimiento deben tener presentes esas funciones, a saber:

- Planificar: El proceso de establecimiento de los objetivos con el fin de alcanzar determinados resultados, así como la identificación de las acciones para alcanzarlos. Por ello deben registrarse políticas, estrategias, planes, programas y pronósticos.

- Organizar: El proceso mediante el cual se divide el trabajo a realizar y la coordinación de los resultados con un propósito común. Las entidades deben asegurar el cumplimiento de las etapas que les permitirán la consecución de los resultados esperados.

- Dirigir: Proceso de conducir y coordinar los esfuerzos de las personas que integran las entidades para ayudarlos al logro de las tareas relevantes en la gestión. Dirigir es tomar decisiones y comprende el compromiso de alcanzar objetivos mediante el liderazgo de una persona o de un grupo de ellas.

- Controlar: proceso de supervisión de las actividades y los resultados de la gestión, comparándolos con los objetivos propuestos.

Gráfico $\mathrm{N}^{\circ} 2$

ELEMENTOS DE LA GESTIÓN DE CONOCIMIENTO ORGANIZACIONAL

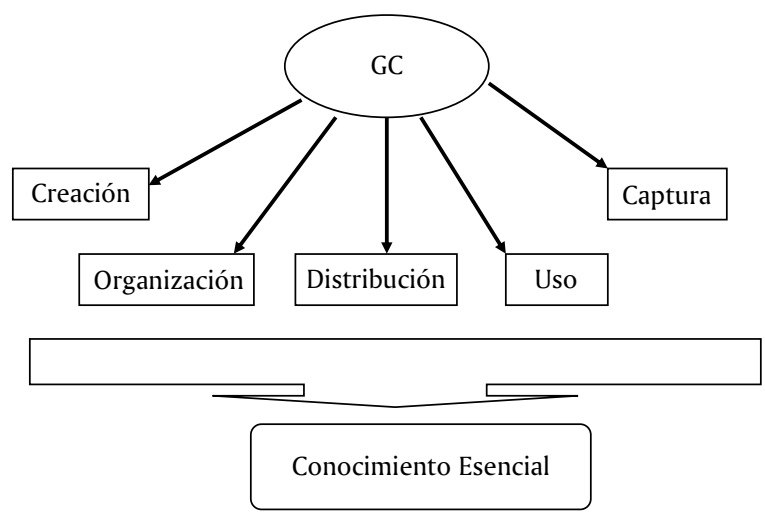

Fuente: Elaboración Propia.

3 Bustelo, Carlota.(2000).

4 Alavi, M. (2000). 
Al valorar cada una de estas funciones debe tenerse en cuenta que el objetivo esencial de la gestión del conocimiento es asegurar fundamentalmente dos procesos: la creación y la transmisión de conocimiento.

En el desarrollo de la gestión de conocimiento es imprescindible el establecimiento de "una política científica", entendida ésta como proyectos y estrategias científicas y tecnológicas de largo aliento, articuladas a estrategias sociales más amplias. Esta política es la que puede marcar realmente el rumbo, los fines, mientras que la gestión proporciona los medios que nos ayudan a alcanzar esos fines. Sin política, la gestión es ciega y no discute rumbos; sin gestión, la política deviene retórica, tal como dice Albornoz ${ }^{5}$.

Cuando hablamos de gestión de conocimiento, según Núñez Jover ${ }^{6}$ debemos entender que esa gestión se define dentro de una política del conocimiento que no es otra cosa que la estrategia global de desarrollo de un territorio.

\section{Componentes necesarios para la gestión de conocimiento.}

Para llevar a cabo la gestión exitosa del conocimiento se requiere de un marco o entorno de trabajo que esté compuesto por los elementos que se muestran en el siguiente gráfico:

Como se observa en el gráfico anterior, el marco de la GC está compuesto por un conjunto

\section{Gráfico No 3}

\section{COMPONENTES NECESARIOS PARA EL ÉXITO DE} LA GC

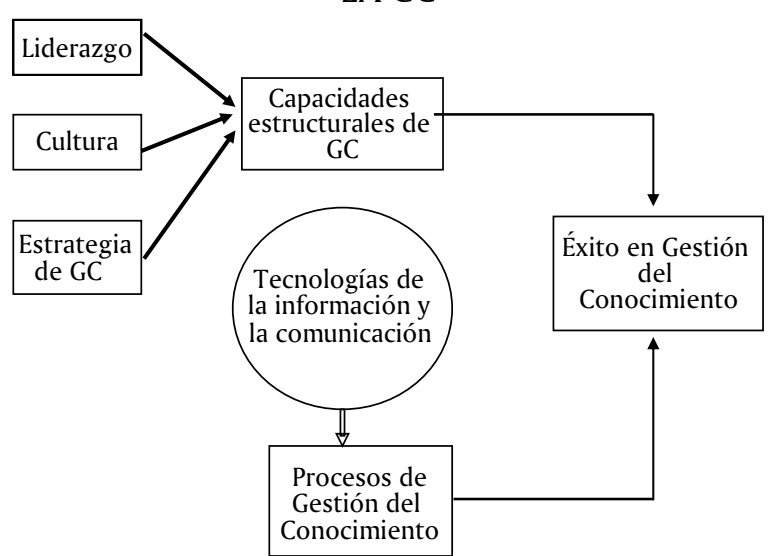

Fuente: León, R. G. (2006). de elementos de carácter organizacional y otros de carácter tecnológico. Según Mohamed (2003), mayor peso tienen los de carácter organizacional, por lo que no es posible pensar en la GC si ésta no se incorpora a la cultura de la entidad.

No obstante, los elementos tecnológicos como las TIC también son importantes aunque no de forma directa, sino a través de su introducción y utilización en los procesos que se definan para esta gestión. A medida que se investiga en este tema se tiene más certeza de que la esencia de la gestión de conocimiento son las personas.

El rol o función que cumplen las tecnologías de la información y las comunicaciones como herramienta es facilitar la conservación y almacenamiento del conocimiento, su organización y categorización, así como brindar las posibilidades para compartirlo, pero sobre todo el de acelerar la velocidad de transferencia de dicho conocimiento.

Existen diferentes tecnologías que están enfocadas en la generación, codificación y transferencia de conocimiento; por la importancia que tienen para este trabajo detallaremos algunas de ellas.

- Sitios web: Este tipo de tecnología ofrece enlaces a sitios importantes, brinda información específica de un tema en particular o presenta contenidos para intercambiar información, y facilita búsquedas de información avanzada; podrían denominarse Portales de Conocimiento.

El sitio web es una herramienta fundamental para la transversalidad de las áreas que pertenecen a una organización, facilitando la integración. La particularidad única de un sitio web es la posibilidad de hacer converger gran cantidad de información en un solo espacio y con un acceso temporal imposible de conseguir por otros medios.

- Intranet: Las intranets representan un ambiente de tecnología heterogénea que conecta diferentes plataformas de hardware, ambientes de sistema operativo e interfaz de usuario con el fin de permitir la comunicación ininterrumpida, la colaboración y transacciones e innovación.

\footnotetext{
5 Albornoz. (1997).

6 Núñez Jover. (2006).
} 
Las intranets son medios para compartir datos dentro de una comunidad concreta. Son flexibles y se adaptan a las necesidades de cada comunidad; posibilitan un rápido acceso y procesamiento de información. Permiten así mismo definir diferentes dominios lógicos de seguridad, ofreciendo una mayor o menor accesibilidad y transparencia.

De esta forma la intranet representa un importante vínculo entre la organización y sus trabajadores. La esencia de ellas radica en crear espacios virtuales y permitir -de forma dinámica- compartir, intercambiar y difundir información, recursos y conocimiento.

Desde el punto de vista del Espiral del Conocimiento visto en el Gráfico № 1, la intranet proporciona un entorno específico para codificar y transferir el conocimiento.

- Los entornos virtuales de aprendizaje. Son vistos desde el punto de vista de Dillenbourg ${ }^{7}$ como un espacio social en el que existe interacción social sobre la información mediante la tecnología, incluyendo actividades sincrónicas (como el chat) y actividades asincrónicas (como por ejemplo el correo electrónico y los foros), presentando además diferentes tipos de comunicación uno a uno, uno a muchos o muchos a muchos, y comunicación indirecta tal como la que se genera al compartir objetos. Son un importante medio para organizar, compartir y generalizar conocimiento.

\section{Funcionalidades que han de cumplir las TIC que dan soporte a la gestión de conocimiento.}

Las TIC que dan soporte a la GC deben cumplir un conjunto de funcionalidades para garantizar que ésta sea efectiva. A continuación se describen aquellas que los autores de este trabajo consideran como principales (ver Cuadro №1).

\section{Estrategia de gestión de conocimiento en las organizaciones.}

De acuerdo a lo valorado, en el proceso de gestión de conocimiento se deben tener en cuenta las funciones de planificación, organización, dirección y control; ninguna de estas funciones se pueden aplicar al proceso de GC en las condiciones actuales del desarrollo de las TIC en las diferentes entidades si no se cuenta con una

\section{Cuadro $\mathbf{N}^{\mathrm{o}} 1$}

Resumen de las funcionalidades que deben cumplir las TIC que dan soporte a la gestión de conocimiento.

\begin{tabular}{|c|c|}
\hline $\begin{array}{c}\text { Arquitectura } \\
\text { de información }\end{array}$ & $\begin{array}{l}\text { Se encarga de la organización de la infor- } \\
\text { mación (clasificación y etiquetado), dise- } \\
\text { ño de los sistemas de recuperación de } \\
\text { la información (sistemas de navegación, } \\
\text { de búsqueda y orientación), y diseño } \\
\text { de la estructura de la información y la } \\
\text { interacción (definición de los procesos } \\
\text { de interacción con el sistema). }\end{array}$ \\
\hline Usabilidad & $\begin{array}{l}\text { Se encarga de velar por la facilidad de } \\
\text { uso del sistema mediante el conocimien- } \\
\text { to experto, la aplicación de estándares } \\
\text { (ISO, buenas prácticas) y la observación } \\
\text { y empatía con el usuario. }\end{array}$ \\
\hline $\begin{array}{l}\text { Diseño } \\
\text { centrado en el } \\
\text { usuario }\end{array}$ & $\begin{array}{l}\text { Supone la inclusión del enfoque del } \\
\text { usuario en todas las fases del ciclo de } \\
\text { vida del sistema, mediante la investi- } \\
\text { gación continua de sus necesidades y } \\
\text { valoraciones. }\end{array}$ \\
\hline Categorización & $\begin{array}{l}\text { Modo de clasificar la información recu- } \\
\text { rriendo a la división del todo en partes } \\
\text { más manejables. Esta categorización } \\
\text { puede ser alfabética, cronológica, fun- } \\
\text { cional, etc. }\end{array}$ \\
\hline Búsqueda & $\begin{array}{l}\text { Esta funcionalidad puede verse aplicada } \\
\text { en diversos formatos (índices, motores } \\
\text { de búsqueda, directorios, etc.), pero } \\
\text { su objetivo es que el usuario pueda } \\
\text { encontrar aquel material que necesita } \\
\text { de manera más sencilla. }\end{array}$ \\
\hline $\begin{array}{c}\text { Edición y } \\
\text { distribución }\end{array}$ & $\begin{array}{l}\text { Posibilidad de editar contenido, guardar } \\
\text { ese contenido y ponerlo a disposición } \\
\text { de los demás. Esta funcionalidad lleva } \\
\text { añadidas otras características como son } \\
\text { la utilidad, la actualización y el almace- } \\
\text { namiento. }\end{array}$ \\
\hline $\begin{array}{l}\text { Soporte de } \\
\text { procesos }\end{array}$ & $\begin{array}{l}\text { Posibilidad de realizar diversos procesos } \\
\text { de utilidad para los usuarios y la organi- } \\
\text { zación (consulta de información diaria, } \\
\text { envío de mensajes, etc.). }\end{array}$ \\
\hline Personalización & $\begin{array}{l}\text { Posibilidad de que los usuarios orga- } \\
\text { nicen la información de acuerdo a sus } \\
\text { perfiles; esto repercute directamente en } \\
\text { la facilidad de procesar la información. }\end{array}$ \\
\hline Presentación & $\begin{array}{l}\text { Capacidad del sistema de presentar } \\
\text { la información de manera eficiente e } \\
\text { intuitiva, teniendo en cuenta que la } \\
\text { Intranet es el único punto de acceso a } \\
\text { la información. }\end{array}$ \\
\hline $\begin{array}{l}\text { Facilidad de } \\
\text { aprendizaje }\end{array}$ & $\begin{array}{l}\text { Constituye uno de los puntos más fuer- } \\
\text { tes para la implantación de la GC dentro } \\
\text { de la organización. El éxito de implan- } \\
\text { tación del sistema tecnológico de GC } \\
\text { es proporcional al grado de facilidad de } \\
\text { aprendizaje de la tecnología utilizada. }\end{array}$ \\
\hline
\end{tabular}

7 Dillenbourg. (2000). 
estrategia coherente para la acción. La estrategia de una entidad incluye los movimientos y enfoques que diseña la gestión tomando en cuenta las condiciones del entorno, para conseguir resultados relevantes que se puedan usar y aplicar en la solución de los problemas que demanda la ciencia y la técnica. Ello presupone que la gestión del conocimiento en las entidades parte de una dirección estratégica unida a su visión, su misión y la determinación de los objetivos.

Entre los principales aspectos a atender como parte de una estrategia de gestión de conocimiento ${ }^{8}$ se pueden destacar:

- La creación de comunidades de conocimiento por ramas afines del saber e interdisciplinarias, que garanticen la relación humana necesaria para garantizar un flujo eficaz de conocimientos.

- Disponer de una intranet eficiente para el intercambio de información, conocimientos y experiencias.

- Actualización sistemática de la información necesaria para agilizar y hacer más efectiva la toma de decisiones.

- Disponer de Bases de Datos que beneficien a estudiantes, profesores, investigadores y expertos.

- Gestionar el conocimiento tácito y convertirlo en conocimiento explícito.

- Disponer de un repositorio de información de conocimiento al que tengan acceso todos los trabajadores, complementado con herramientas de comunicación para intercambiar experiencias, conocimientos y estudio de casos, lo que implicará una mejora en los procesos de gestión.

Otro elemento importante es lo que hoy se ha dado en llamar cultura organizacional. Las entidades tienen una cultura organizacional propia y reconocida en el sistema de valores, ideas, hábitos $\mathrm{y}$ tradiciones compartidos por las personas que integran la organización, y todos esos elementos tienen que ponerse en función de la estrategia y los objetivos trazados por la entidad que gestiona el conocimiento. Aún cuando siempre exista una cierta resistencia al cambio, lo cierto es que dicha cultura organizacional tendrá una influencia signi- ficativa en la gestión; por lo tanto, las entidades deben evaluar siempre las fuentes generales que promueven la resistencia y las acciones necesarias para que esta pueda ser vencida.

\section{CONCLUSIONES}

1. La gestión del conocimiento se perfila en la actualidad como un componente estratégico de gran trascendencia en toda organización que pretenda destacar en el sector donde se desarrolla.

2. Los aportes asociados a la gestión del conocimiento van más allá de la contribución al desempeño exitoso en el plano económico. La técnica y las herramientas permiten a la dirección de las organizaciones conocer realmente los valores intelectuales bajo su mando y prepararse para el futuro, no sólo sobre la base de los valores materiales y financieros disponibles, sino también sobre la base de los valores intelectuales que dirige.

3. La gestión del conocimiento identifica y explota el conocimiento creado en la organización y el adquirido en el trabajo cotidiano; generaliza las mejores prácticas, propicia el incremento del capital intelectual de la organización y su valor (de mercado o social), a la vez que facilita la generación de nuevos conocimientos y su materialización en productos y servicios.

4. Cualquiera que sea la estrategia a seguir existen diversas herramientas que pueden ser el medio para alcanzar una gestión de conocimiento acorde a los objetivos de la organización. No existe una única herramienta que lo haga todo, es necesario conjugar varias de ellas para obtener una mayor ventaja competitiva.

\section{BIBLIOGRAFÍA}

Barnes G. J. (2000). Su empresa ¿De clase mundial? La Habana, Editorial Academia.

Castro Díaz-Balart, Fidel. (2004). Ciencia, Tecnología y Sociedad Científico-Técnica. La Habana.

Darín, S. (2006). "El impacto de las nuevas tecnologías de la información y las comunicaciones. Sociedad del conocimiento, educación y democracia”. En: CD ROM La Universalización de la Universidad por un mundo mejor. Evento Universidad 2006. ISBN: 959-0282-08-3.

8 Estrada, V. (2006). 
Estrada, V. y Benítez, F. (2006). "La Gestión de conocimiento en la nueva universidad cubana". En: La nueva universidad cubana y su contribución a la universalización del conocimiento. Colectivo de autores. La Habana, Editorial Félix Varela.

Nonaka, Ikujuro and Takeuchi, Hirotaka. (1995). The knowledge creating company. How Japanese companies create the dynamics of innovation. New York, Oxford University Press.

Núñez J. Jorge. (2004). La ciencia y la tecnología como procesos sociales: Lo que la educación científica no debería olvidar. La Habana, Ciencias Sociales.
Nuñez, J. y otros. (2006). "La gestión del conocimiento, la ciencia, la tecnología y la innovación en la nueva universidad: una aproximación conceptual". En: La nueva universidad cubana y su contribución a la universalización del conocimiento. Colectivo de Autores. La Habana, Editorial Félix Varela.

Reyes, C. (2004). "Una breve introducción a las tecnologías de la información para la gestión del conocimiento". En: Intangible Capital. № 4. ISSN: 16979818 (Cod:0026). 